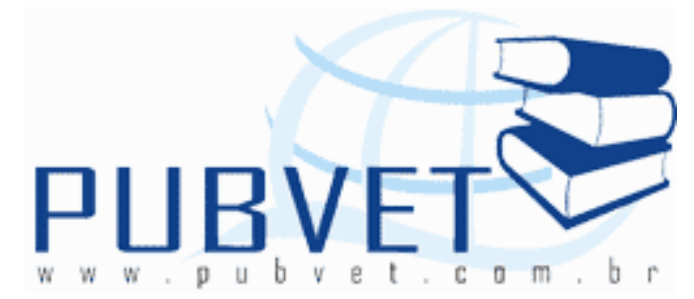

PUBVET, Publicações em Medicina Veterinária e Zootecnia.

\title{
O caminho da nanotecnologia na produção animal brasileira
}

\section{Edson Sadayuki Eguchi ${ }^{1}$, Ulysses Cecato $^{2}$ e Samuel Laudelino Silva ${ }^{1}$}

${ }^{1}$ Prof. Msc. Dep. Zootecnia, Universidade do Estado de Mato Grosso (UNEMAT).

${ }^{2}$ Prof. Dr. Dep. Zootecnia, Universidade Estadual de Maringá (UEM).

\section{Resumo}

A "nanotecnologia" possibilita a construção de materiais ou estruturas, a partir dos próprios átomos na escala manométrica $\left(\mathrm{nm}=10^{-9} \mathrm{~m}\right)$. Atualmente as áreas mais estudadas e divulgadas pelas pesquisas em nanotecnologia estão ligadas aos setores de medicina, metalurgia, condutores e informática. E, no futuro, permitirão a convergência dessas novas tecnologias esteja voltada para outras áreas como das ciências agrárias em produção animal. Neste contexto, o objetivo do presente artigo é trazer informações relativas da nanotecnologia com possibilidades de aplicação neste setor. Assim, por meios da nanotecnologia pode se obter produtos, como a nova água (Neowater); o nanocarro com possibilidade de transporte de produtos dentro de organismos vivos; "metal amorfo"; microencapsulação; a homeopatia; a produção de nanopartículas metálicas; nanosensores e nanotécnica. O Brasil segue com desenvolvimento de nanosensores e deve se perguntar se estamos preparados com as mudanças de cunho revolucionário que poderão romper o atual paradigma de nossa produção agropecuária.

Palavras-chave: Nanociência, tecnologia, agricultura, pecuária. 


\section{The way of nanotechnology in Brazil animal production}

\section{Abstract}

The "nanotechnology" enables the construction of structures or materials, from the atoms themselves in the gauge scale $(\mathrm{nm}=10-9 \mathrm{~m})$. Currently the areas most studied and published by research in nanotechnology are linked to the sectors of medicine, metallurgy, conductors and computer. And in the future, enable the convergence of these technologies are focused on other areas such as agricultural science in animal production. In this context, the aim of this paper is to provide information relating to nanotechnology application possibilities in this sector. Thus, by means of nanotechnology products can be obtained, as the new water (Neowater); nanocar with the possibility of transporting products within living organisms; "amorphous metal"; microencapsulation; homeopathy, the production of metal nanoparticles, nanosensors and nanotécnica. Brazil follows with development of nanosensors and must wonder whether we are prepared to die revolutionary changes that could break the current paradigm of our agricultural production.

Keywords: Nanoscience, technology, agriculture, livestock.

\section{INTRODUÇÃO}

A palavra "nanotecnologia" está associada com tecnologias que possibilitam a construção de materiais ou estruturas, a partir dos próprios átomos em uma escala extremamente reduzida aos nossos sentidos: a do nanômetro $(\mathrm{nm})$, uma subunidade do metro e que corresponde à bilionésima parte do metro $\left(10^{-9} \mathrm{~m}\right)$ (RIBOLDI, 2009). Dessa forma, significa a habilidade de manipulação de átomo por átomo na escala compreendida entre $0,1 \mathrm{e}$ $100 \mathrm{~nm}$ buscando uma nova organização estrutural para fins principalmente comerciais (OLIVEIRA, 2009).

A nanotecnologia ganhou importância significativa no final do século $X X$ apoiando-se no fato de que as propriedades dos materiais (óticas, elétricas, 
magnéticas, catalíticas, etc) dependem fortemente do tamanho das partículas destes materiais. Mas as primeiras idéias foram apresentadas no final do ano de 1959 por Richard Feynman, Prêmio Nobel em Física, na qual um dia seria possível manipular átomos individualmente. Segundo Rai et. al., (2009), a criação e utilização da palavra "nanotecnologia" foi em 1974, proposta por Norio Taniguchi, da Universidade de Tókio, onde fez a distinção na engenharia em escala além do micrométro.

Nos anos 82 com o desenvolvimento dos chamados, microscópios de varredura por sonda patenteada pela IBM, tornou-se possível mapear as superfícies de objetos de dimensões nanométricas, por meio de uma agulha muito fina, construindo uma imagem com resolução em escala atômica (um átomo, menor unidade possível de um elemento químico) (Ramos et. al, 2010). Dentre os microscópios de varredura por sonda, destacam-se os de tunelamento, de força atômica e de campo, que permitiram avanços relativos á manufatura molecular e atômica.

Segundo Riboldi (2009), esses microscópios, possuem uma ponta de dimensões ínfimas (constituída de poucos átomos) que pode ser aproximada e "varrer" uma superfície de modo a "sentir imperfeições" de até 0,1 Angstron, possibilitando "ver os átomos". Além disso, quando são aplicados valores apropriados de tensão nessas pontas, um único átomo pode ser arrancado de um ponto e depositado em outro.

Com definições igualmente comparáveis a palavra tecnologia e a ciência (OLIVEIRA, 2009). Atualmente a nanotecnologia e a nanociências vêm dando um impulso extraordinário à inovação e evolução do conhecimento, permeando as principais áreas de Física, Biologia e Química. Possibilitando assim, a convergência de novas tecnologias para outras áreas especifica como a das ciências agrárias envolvendo a biotecnologia, a informática de campo, a neurociência, a ciência genômica, entre outras.

Conforme Opara (2004), a adoção e prática da "agricultura inteligente" significará uma mudança radical do paradigma de produção, com a adoção desse tipo de tecnologia que provocará dolorosos impactos tecnológicos e 
socioeconômicos. O potencial da nanoeletromecanização na agricultura moderna será imenso. Como decorrência, surgirão questões sociais, políticas e éticas a serem enfrentadas com 0 desenvolvimento do processo de miniaturização e eletromecanização da agricultura (nanoagricultura).

Prevê ainda, que um dos campos mais promissores denominada de agrinfortronics, que poderia ser traduzido para "agroinfortrônica", ou seja, a união de agricultura, informática e eletrônica é o da identificação, captação, análise, armazenamento e transmissão de informações precisas e confiáveis sobre a produção/manejo ambiental de animais/plantas de modo a atender às demandas por elevadas produções e boa qualidade dos produtos.

Nos países do Primeiro Mundo as possíveis aplicações diretas das nanotecnologias aos setores agrícolas e pecuárias são bem menos estudadas e divulgadas quando comparadas a setores como medicina, metalurgia, condutores, informática, etc. No Brasil foi lançado em 2006 o Laboratório Nacional de Nanotecnologia para o Agronegócio da Embrapa, em São Carlos, onde poderemos esperar desenvolvimentos focados no setor, principalmente de nanosensores de monitoramento de qualidade alimentar e deteç̧ões de doenças em animais. Isto significa que os agricultores brasileiros que hoje adotam sistemas de produções convencionais, orgânicos, agroecológicos, utilizam sementes originadas da engenharia genética ou qualquer outra forma de produzir e comercializar, desconhecem quase totalmente os avanços já alcançados pelas nanotecnologias aplicadas ao setor rural, e muito menos as possíveis consequências econômicas, sociais, ambientais e políticas. Neste contexto, o objetivo do presente artigo é trazer informações relativas da natureza da nanotecnologia com possibilidades de aplicação na produção animal.

\section{NANOTECNOLOGIA NA PRODUÇÃO ANIMAL}

O Brasil com seus 59 programas de desenvolvimento, em 13 áreas estratégica sendo uma envolvendo a área de nanotecnologia, que 
representaram no período 2007-2009, 68\% das atividades do Plano de Ação em Ciência, Tecnologia e Inovação para o Desenvolvimento Nacional (PACTI), com recursos financeiros do Ministério da Ciência e Tecnologia (MCT), foram cerca de R\$ 3 bilhões (Rezende, 2011).

Os fundamentos tecnológicos da maior parte das atividades agrícolas brasileiras dependem dos denominados insumos modernos. Esse sistema de produção baseia-se no uso de agroquímicos, motomecanização, irrigação, hormônios de crescimento, antibióticos, entre outros. A natureza da nanotecnologia molecular permite inferir na capacidade produtiva, em conjunto com outras tecnologias, alterar drasticamente as históricas características da agricultura. A incerteza dos resultados e riscos, que forçosamente se enfrentam nos atuais processos de produção agrícola no contexto de um ambiente natural, praticamente deixaria de existir e/ou seria minimizada ao se utilizarem as nanotecnologias (Ramos et. al, 2010).

Segundo Riboldi (2009), atualmente existem as três seguintes abordagens distintas para a nanotecnologia: 1. Abordagem "Top-down" (de cima para baixo) e relacionada com a construção de dispositivos por desgaste de materiais macroscópicos, que é utilizada em microeletrônica, para produção de "chips" de computadores. 2. Formação espontânea de dispositivos, a partir de seus componentes moleculares, utilizando técnicas tradicionais de Química e de Ciências dos Materiais. 3. "Botton-up" (de baixo para cima) que possibilita a construção de estruturas átomo por átomo ou molécula por molécula, só disponível com avanços e aperfeiçoamentos tecnológicos uma vez que necessita de controle muito rigoroso e fino da matéria.

A exemplo de que toda nanotecnologia desenvolvida especificamente para um setor, também servirá de alguma maneira na aplicação relativa na produção animal em forma de monitoramento ou mesmo de aplicação direta na saúde, na alimentação, controle de parasitas, conforto térmico e ambiental desses animais (Mella \& Mendo, 2010). Abaixo seguem alguns itens de importância com aplicações diretas ou mesmo indiretos em um futuro próximo na produção animal. 


\section{1. Água.}

A água é a base para a nossa sobrevivência, batizada de Neowater, para produzir a nova água, são utilizadas nanopartículas inertes, aquecidas e lançadas em um recipiente de água normal a uma temperatura de $4^{\circ} \mathrm{C}$. A seguir, a solução é irradiada com ondas de radiofreqüência, causando uma onda de choque que faz com que a nanopartícula se divida. O processo continua, e cada pedaço divide-se novamente, e assim por diante, em um processo que os cientistas chamaram de "Nano Bang". O resultado é a geração de nanopartículas cada vez menores, o que gera uma área superficial gigantesca. É este o efeito que imita a condição encontrada no interior das organelas, que provavelmente é o responsável pelo comportamento físico diferenciado da água intracelular. A nova água mostrou-se altamente eficaz contra a propagação da bactéria Bacillus subtilis. Ela também pode substituir diversos solventes, como o álcool. Não se sabe explicar por que a água intracelular (aquela que está no interior das nossas células) é diferente de todas as outras formas de água. Ao conseguir produzir água com as mesmas propriedades da água intracelular, abre-se caminho para uma nova fase na pesquisa de medicamentos, já que ela permite, entre outras possibilidades, a solubilidade de fármacos hidrofóbicos e a estabilização de proteínas e culturas celulares (Riboldi, 2009).

\subsection{Nanocarro.}

Em 2005 cientistas da Universidade Rice, Estados Unidos construíram o menor carro do mundo, construído com moléculas que precisava ser aquecido a $200^{\circ} \mathrm{C}$ para andar por isso considerado de nanocarro a quente. A temperatura ambiente, o nanocarro fica totalmente grudado sobre a superfície de ouro na qual ele foi construído. Mas, a cerca de $200^{\circ} \mathrm{C}$, ele fica livre e pode andar. Para provar que o nanocarro está realmente andando, e não escorregando sobre a superfície, os cientistas fizeram imagens a cada minuto e 
analisaram o movimento. "A síntese e teste de nanocarros e outras máquinas moleculares está oferecendo elementos críticos em nossas investigações da fabricação molecular de 'baixo-para-cima'", explica James M. Tour, um dos coordenadores da pesquisa. "Nós eventualmente iremos querer mover objetos e trabalhar de forma controlada em escala molecular e esses veículos são excelentes balões de ensaio para isso. Eles estão nos ajudando a aprender as regras básicas".

\subsection{Nanomateriais (Metais amorfos) e nanopartículas.}

Um processo de fabricação de peças minúsculas que utiliza o chamado "metal amorfo" poderá revolucionar a fabricação de nanomáquinas e qualquer outra estrutura em nanoescala, desde memórias de computador até sensores biomédicos para uso no interior do corpo humano.

$\mathrm{O}$ nanomaterial fotocatalisador $\mathrm{TiO}_{2}$, principalmente na sua forma anatasse, é utilizado na fotodegradação de compostos orgânicos no tratamento da água. Preso sobre diferentes superfícies como polímeros, vidros e metais evitando a sua saída com os efluentes (Li et al., 2006).

Pesquisadores Rohner et al. (2007) desenvolveram nanopartículas altamente disponíveis de fosfato férrico, provando que a fonte esta em nanoescala pode aumentar seu valor nutricional na alimentação animal.

As nanopartículas de prata foram testadas sem sucesso (com objetivos antibacterianos) em embriões de ovos de galinha. Embora eles não afetassem o desenvolvimento do embrião, reduziu o número e tamanho dos folículos linfóides (Grodzik \& Sawosz, 2006).

Nanopartículas de carbono e nanofibras, são atualmente os mais atrativos devido a suas diferentes formas. A nanopartícula do terceiro alótropo do carbono, fulereno $\left(\mathrm{C}_{60}\right)$, é a mais pesquisada e produzida em grande escala atualmente. Partículas de nanofulereno (nano- $\mathrm{C}_{60}$ ) vêm sendo testadas para atuarem como sensores e catalisadores e, devido a sua estrutura única, podem 
também ser usadas para liberação controlada de fármacos (Paschoalino et al., 2010).

\subsection{Encapsulamento de componentes.}

Um caso bastante concreto e impressionante de aplicação na agricultura é a formulação de insumos em nanoescala, que implica seu encapsulamento, ou seja, envolver o ingrediente ativo em nanoescala com uma espécie de minúsculo "envelope" ou "concha". Inclui-se nessa tecnologia a possibilidade de se controlarem as condições nas quais o princípio ativo deve ser liberado diretamente nas plantas. Segundo as indústrias, as vantagens da microencapsulação de pesticidas são evidentes, pois permitem: que tamanho reduzido das partículas otimize sua eficácia; que cápsulas sejam programadas para liberar seu princípio ativo em certas condições; etc., (Ramos et. al, 2010).

\subsection{Nanotecnologia e Homeopatia}

Muito popular a homeopatia trata seres humanos, animais, e plantas, de forma nanotecnológica proporcionando o aumento de imunidade e resistência de doenças oportunistas, nas quais os pesquisadores apontam nova tendência nanotecnológica de tratamento (Souza, 2002). A nova tendência reduz o impacto industrial sobre o planeta, devido à eficiência na utilização da energia, uma vez que, assim como a nanotecnologia, à homeopatia também utiliza-se de átomos e energia.

\subsection{Nanotecnologia e microbiologia}

Grande parte das pesquisas sobre a síntese extracelular de fungos de nanopartículas esta sendo realizado com prata (Rai et. al., 2009). Fungos têm certas vantagens no número de síntese de nanopartículas em comparação com 
outros organismos, nomeadamente eles são relativamente fáceis de isolar em cultura e secretam grandes quantidades de enzimas extracelulares (Mandal et al., 2006). Alguns das nanopartículas que podem ser produzidos por fungos, podem apresentar no futuro uma relevância de tecnologias emergentes nas áreas farmacêuticas e no controle de infecções. A produção de nanopartículas metálicas por fungos parece ser um processo relativamente biotecnológico simples, predominantemente envolvendo apenas a reação de filtrados de cultura de fungos em soluções de sais metálicos.

\subsection{Nanosensores de monitoramento.}

Nanosensores feitos de nanotubos de carbono, ou os nanocantilevers (estruturas de braços em balanço/equilíbrio), são pequenos o suficiente para detectar e medir as proteínas individualmente, ou mesmo moléculas. Nanopartículas ou nanosuperfícies podem ser engenheiradas para disparar um sinal elétrico ou químico em presença de um contaminante, tal como uma bactéria. Outros nanosensores funcionam disparando uma reação enzimática ou utilizando moléculas nanofabricadas, chamadas dendrímeros, que se ramificam como sonda para se ligarem a substâncias químicas e proteínas alvo (ETC Group, 2004).

Exemplo da "língua eletrônica", desenvolvida por Luiz Henrique Mattoso e equipe na Embrapa, dispositivo que alia sensores químicos de espessura nanométrica com um sofisticado programa de computador para detectar sabores, qualidades, contaminantes em produtos agrícolas.

\subsection{Micro e Nanofluídicos.}

A microfluídica está sendo utilizada na reprodução de animais para classificar fisicamente esperma e óvulos. Líder nesse campo é a XY Inc., do Colorado (EUA), que está usando uma técnica microfluídica chamada de citometria de fluxo, para segregar espermatozoides com características 
masculinas ou femininas para fins de seleção de sexo. A XY conseguiu criar cavalos, gado, ovelhas e porcos com escolha de sexo, e atualmente disponibiliza tecnologia para criadores comerciais. A Arrynx, uma empresa nova em nanotecnologia, que desenvolveu um novo sistema microfluídico chamado MatRyx, utiliza uma nanotécnica na qual diminutos raios laser de arrasto capturam espermatozóides individuais e depois os classificam por peso. O MatRyx pode classificar cerca de 3000 espermatozóides por segundo, e visa a comercialização para a reprodução de gado (ETC Group, 2004).

\section{Brasil e o futuro da nanotecnologia}

A produção ou o desenvolvimento de nanomateriais gera nanopartículas que podem ser potencialmente perigosas para o ser humano e para o meio ambiente, uma vez que flutuam facilmente e podem se deslocar por grandes distâncias (Riboldi, 2009).

O Brasil é um dos países que fez parte da ISO/TC 229 (ISO/Technical Committee) para elaboração de protocolos padrão no período de 2005 a 2010 para a certificação ISO em nanomateriais (Paschoalino et al., 2010).

Segundo Raupp (2012) a PORTARIA No 245, DE 5 DE ABRIL DE 2012., Institui o Sistema Nacional de Laboratórios em Nanotecnologias - SisNANO como um dos elementos do Programa Nacional de Nanotecnologia, no âmbito da Estratégia Nacional de Ciência, Tecnologia e Inovação e associado ao Plano Brasil Maior. O Ministro de Estado da Ciência, Tecnologia e Inovação, no uso das atribuições que the confere o art. 87, parágrafo único, inciso II, da Constituição Federal, resolve: Art. $1^{\circ}$ Fica instituído o Sistema Nacional de Laboratórios em Nanotecnologias - SisNANO, como um dos elementos do Programa de Nacional de Nanotecnologias, no âmbito da Estratégia Nacional de Ciência, Tecnologia e Inovação - ENCTI e associado ao Plano Brasil Maior PBM.

Algumas empresas no Brasil, sozinhas ou em parceria com universidades e institutos de pesquisa dentro e fora do país, têm investido no 
desenvolvimento de produtos e processos de base nanotecnológica. O exemplo segundo Raupp (2012) a PORTARIA No 117, DE 13 DE FEVEREIRO DE 2012. Institui o Centro Brasil-China de Pesquisa e Inovação em Nanotecnologia CBC-Nano. O Ministro de Estado da Ciência, Tecnologia e Inovação, no uso das atribuições que lhe são conferidas pelo art. 87, parágrafo único, incisos I e II, da Constituição Federal, e pelo Decreto no 5.886, de 6 de setembro de 2006, resolve: Art. $1^{0}$ Fica instituído o Centro Brasil-China de Pesquisa e Inovação em Nanotecnologia - CBC-Nano, na forma de uma rede cooperativa de pesquisa e desenvolvimento, como mecanismo de implementação do Acordo sobre Cooperação Científica e Tecnológica firmado por ambos os países, no âmbito dos objetivos estratégicos nacionais na área de nanotecnologia.

\section{CONCLUSÕES}

O mundo caminha para uma nova revolução na concepção nano e o Brasil, ao longo de 20 anos de trabalho embora não apresentem bons resultados diretos na produção animal em nanociência e nanotecnologia, já demonstram potencial de crescimento, associados a capitação tecnológicas indiretas de outras áreas mais especificas.

As nanotecnologias oferecem oportunidades para toda uma sociedade o que atualmente se percebe é uma corrida pela busca da conquista de difusão desta tecnologia, mas elas também podem trazer profundos riscos sociais e ambientais quando manipulados sem normas técnicas, mesmo em áreas distantes de suas conquistas.

\section{REFERÊNCIAS BIBLIOGRÁFICAS}

ETC GROUP. A invasão invisível do campo. O impacto das nanotecnologias na alimentação e na agricultura. Tradução: José F. Pedros. Revisão técnica: Maria José Guazzelli e Flavio Borghetti. Ottawa: ETC Group, 2004. Disponível em: <http://www.etcgroup.org/documents /NR_DownFarm_final.pdf>. Acesso em: 2 abril 2012. 
GRODZIK, M.; SAWOSZ, E. The influence of silver nanoparticles on chicken embryo development and bursa of Fabricius morphology. Journal of Animal and Feed Sciences. 15(suppl.1): 2006. p.111-114.

Li, G.; Qu, J.; Zhang, X.; Ge, J.; Water Res. 2006, vol.40, p213.

MANDAL, D.; BOLANDER, M.E.; MUKHOPADHYAY, D.; et al. The use of microorganisms for the formation of metal nanoparticles and their application. Applied Microbiology and Biotechnology 69, 2006. p.485-492.

MARTINS, P.R. Nanotecnologia, sociedade e meio ambiente. São Paulo: Xamã, 2006.p 344.

MELLA, M.R.; MENDO, O.H. Nanotechnology on animal production. Tropical and Subtropical Agroecosystems. 12 (2010). p.423-429.

OLIVEIRA, D.M.F.de. Síntese e caracterização de óxidos metálicos nanoestruturados e sua utilização em nanocompósitos com poli (álcool vinílico). 2009. 153f. (Doutorado em Química) - Universidade Estadual de Maringá, Maringá.

OPARA, L.U. Emmerging technological innovation triad for smart agriculture in the 21th century. Parte I. Prospects and impacts of nanotechnology in agriculture. Oman, 2004.

PASCHOALINO, M.P.; MARCONE, G.P.S.; JARDIM, W.F. Os nanomateriais e a questão ambiental. Quím. Nova. vol.33 nº.2. São Paulo. 2010.

RAI, M., YADAV, A.; BRIDGE, P. et. al. Myconanotechnology: a New and Emerging Science. Applied mycology / edited by Mahendra Rai and Paul Dennis Bridge. P.258-267. 2009.

RAMOS, S.de F.; MARTINS, P. R.; DULLEY, R. D.; et. al. Os impactos das nanotecnologias na cadeia de produção da soja. Informações Econômicas, SP, v.40, n.6, jun. 2010. p 45-55.

RAUPP, M.A. Ministério da Ciência, Tecnologia e Inovação. PORTARIA No 245, DE 5 DE ABRIL DE 2012. . Diário Oficial da União. N68. 2012.5p. (http://www.in.gov.br/imprensa/ visualiza/index.jsp? jornal=1\&pagina $=5 \&$ data $=09 / 04 / 2012)$.

RAUPP, M.A. PORTARIA No 117, DE 13 DE FEVEREIRo De 2012. Institui o Centro BrasilChina de Pesquisa e Inovação em Nanotecnologia - CBC-Nano. Diário Oficial da União. Sessão1. N.32. 2012. 5p. (http://www.in.gov.br/imprensa/visualiza/index.jsp?jornal=1\& pagina $=5 \&$ data $=14 / 02 / 2012$ )

REZENDE, S.M. Produção científica e tecnológica no Brasil: conquistas recentes e desafios para a próxima década. Rev. adm. empres. vol.51 no.2 São Paulo Mar./Apr. 2011. p.202-209.

RIBOLDI, B.M. Nanotecnologia: Fundamentos e Aplicações. Departamento de FísicaInstituto de Geociências e Ciências Exatas Campus de Rio Claro, Universidade Estadual Paulista Júlio de Mesquita Filho. 2009. 22p.

ROHNER, F.; ERNST, F.; ARNOLD, M.; et. al.. Synthesis, characterization, and biodisponibility in rats of ferric phosphate nanoparticles. Journal of Nutrition. vol.137: 2007.p.614-619.

SOUZA, M.F.A. Homeopatia Veterinária. I Conferência Virtual Global sobre Produção Orgânica de Bovinos de Corte. 02 de setembro à 15 de outubro de 2002. http://www.cpap.embrapa.br/ agencia/ congressovirtual/pdf/portugues/02pt02.pdf. 